\title{
Prevalence of mutations in a panel of breast cancer susceptibility genes in BRCA1/2-negative patients with early-onset breast cancer
}

\author{
Kara N. Maxwell, MD, PhD1, Bradley Wubbenhorst, MS², Kurt D'Andrea, BS², Bradley Garman, BS², \\ Jessica M. Long, MS, LCGC'1, Jacquelyn Powers, MS, LCGC', Katherine Rathbun, BS², \\ Jill E. Stopfer, MS, LCGC'1, Jiajun Zhu, BS², Angela R. Bradbury, MD ${ }^{1,3}$, Michael S. Simon, MD, MPH4, \\ Angela DeMichele, MD, MSCE ${ }^{1,3}$, Susan M. Domchek, MD ${ }^{1,3}$ and Katherine L. Nathanson, MD ${ }^{2,3}$
}

\begin{abstract}
Purpose: Clinical testing for germ-line variation in multiple cancer susceptibility genes is available using massively parallel sequencing. Limited information is available for pretest genetic counseling regarding the spectrum of mutations and variants of uncertain significance in defined patient populations.
\end{abstract}

Methods: We performed massively parallel sequencing using targeted capture of 22 cancer susceptibility genes in $278 \mathrm{BRCA1} / 2$-negative patients with early-onset breast cancer (diagnosed at younger than 40 years of age).

Results: Thirty-one patients (11\%) were found to have at least one deleterious or likely deleterious variant. Seven patients (2.5\% overall) were found to have deleterious or likely deleterious variants in genes for which clinical guidelines exist for management, namely TP53 (4), CDKN2A (1), MSH2 (1), and MUTYH (double heterozygote).
Twenty-four patients (8.6\%) had deleterious or likely deleterious variants in a cancer susceptibility gene for which clinical guidelines are lacking, such as CHEK2 and ATM. Fifty-four patients (19\%) had at least one variant of uncertain significance, and six patients were heterozygous for a variant in MUTYH.

Conclusion: These data demonstrate that massively parallel sequencing identifies reportable variants in known cancer susceptibility genes in more than $30 \%$ of patients with early-onset breast cancer. However, only few patients $(2.5 \%)$ have definitively actionable mutations given current clinical management guidelines.

Genet Med advance online publication 11 December 2014

Key Words: cancer susceptibility; early-onset breast cancer; genetic testing; massively parallel sequencing; multiplex panel testing

\section{INTRODUCTION}

Mutations in the breast and ovarian cancer susceptibility genes, $B R C A 1$ and BRCA2, are found in $10-20 \%$ of women with early-onset breast cancer (defined as breast cancer diagnosed at younger than 40 years of age). ${ }^{1}$ In comparison with women with postmenopausal breast cancer, women with early-onset breast cancer have a worse prognosis with increased recurrence rates, rates of distant metastases, and higher overall mortality. ${ }^{1}$ Because BRCA1/2 genetic testing is recommended for all women with breast cancer diagnosed at younger than 40 years of age, ${ }^{2}$ further expansion of genetic testing to other moderateand high-penetrance genes is commonly considered for this group. Furthermore, it has the potential to identify women who may benefit from targeted breast cancer screening and prevention strategies aimed at decreasing morbidity and mortality, as has been demonstrated for BRCA1/2 mutation carriers. ${ }^{3}$

Beyond $B R C A 1 / 2$, rare highly penetrant mutations in the genes CDH1, PTEN, STK11, and TP53 lead to cancer susceptibility syndromes, ${ }^{4}$ for which the National Cancer Care
Network provides guidelines for genetic testing and clinical management. ${ }^{2}$ In addition to these high-risk genes, breast cancer susceptibility is associated with rare moderate-penetrance mutations in an increasing number of genes, such as ATM, CHEK2, and PALB2, which confer an approximately twofold to fivefold increased risk of breast cancer. ${ }^{4}$ Guidelines do not delineate patient populations for which testing for mutations in moderate-risk genes is expected to be beneficial, nor how the information obtained from this testing should be applied in clinical management of cancer risk.

Despite these limitations, clinical testing based on massively parallel sequencing (MPS) is now commercially available for many known or proposed cancer susceptibility genes. ${ }^{5,6}$ Rather than focusing on sequential testing of individual, well-studied genes based on defined clinical characteristics of the patient's personal and family histories, these tests concurrently screen a large number of genes. A lack of data about the cancer risk and penetrance in women carrying these mutations has made the translation of potential life-saving strategies used in $B R C A 1 / 2$

${ }^{1}$ Division of Hematology/Oncology, Department of Medicine, Perelman School of Medicine at the University of Pennsylvania, Philadelphia, Pennsylvania, USA; ${ }^{2}$ Division of Translational Medicine and Human Genetics, Department of Medicine, Perelman School of Medicine at the University of Pennsylvania, Philadelphia, Pennsylvania, USA; ${ }^{3}$ Abramson Cancer Center, Perelman School of Medicine at the University of Pennsylvania, Philadelphia, Pennsylvania, USA; ${ }^{4}$ Karmanos Cancer Institute, Wayne State University, Detroit, Michigan, USA. Correspondence: Katherine L. Nathanson (knathans@exchange.upenn.edu) 
carriers to these women problematic. ${ }^{7.8}$ Whereas frequencies of $B R C A 1 / 2$ mutations are well studied, data are needed regarding the spectrum of variants in the other cancer susceptibility genes in defined patient populations. Here we report data on the frequency and type of variants in a panel of cancer susceptibility genes in BRCA1/2-negative women with early-onset breast cancer.

\section{MATERIALS AND METHODS Patient ascertainment}

The study population was ascertained from academic and community hospital sites within the Penn Cancer Network and the Karmanos Cancer Institute at Wayne State University. ${ }^{9}$ The majority of the patient population (253 patients, 91\%) was ascertained via the Penn Cancer Network, 16 patients (6\%) were from the Karmanos Cancer Institute at Wayne State University, and ascertainment data were not available for 9 patients (3\%). Acquisition of the patient samples was approved by the institutional review boards of the corresponding institutions, and informed consent was obtained from each participant for use of his or her sample in genetic studies. Eligibility criteria for the study were: (i) diagnosis of breast cancer at younger than 40 years of age; (ii) negative $B R C A 1 / 2$ sequencing in a Clinical Laboratory Improvement Amendments-approved laboratory; and (iii) negative personal or family history of ovarian cancer. Analysis for BRCA1/2 large genomic rearrangements was not required, although negative clinical testing was available for $28 \%$ of patients.

\section{DNA library preparation and sequencing}

For each patient, $1 \mu \mathrm{m}$ of constitutional DNA was blunt-ended and ligated with adapter-embedded indexes. DNA quality, fragment size, and concentration were measured with an Agilent 2100 Bioanalyzer (Agilent, Santa Clara, CA). DNA libraries of sufficient quality were pooled before capture to 24-plex and hybridized to a custom-designed Agilent SureSelect target library covering all coding exons and the flanking 10 base pairs of 22 genes. The genes included 20 study genes plus BRCA 1 and $B R C A 2$ and were as follows: (i) high-penetrance breast cancer susceptibility genes (CDH1, PTEN, STK11, and TP53); (ii) genes known to cause other cancer susceptibility (CDKN2A, MLH1, MSH2, MSH6, and PMS2); (iii) genes known or postulated to be moderate-penetrance cancer susceptibility genes (ATM, BARD1, BRIP1, CHEK2, FAM175A, MRE11A, NBN, $R A D 50, P A L B 2$, and $R A D 51 C$ ); and (iv) MUTYH, which leads to autosomal recessive polyposis.

\section{MPS data analysis}

Raw sequencing data were aligned to the hg19 assembly of the human genome using Burrows-Wheeler Aligner for short-read alignment (http://sourceforge.net/projects/bio-bwa/files/). ${ }^{10}$ BAM files were processed with Genome Analysis Toolkit for detection of single-nucleotide variants and insertion/deletion variants (indels; http://www.broadinstitute.org/gatk/download) $)^{10,11}$ and annotated with ANNOVAR (http://www.openbioinformatics.org/annovar/annovar_download.html). ${ }^{10}$ Data were additionally analyzed using Pindel to improve sensitivity for medium-size indels (http:/gmt.genome.wustl.edu/packages/ pindel/ $/{ }^{10}$ and xhmm for large genomic rearrangements (https:// atgu.mgh.harvard.edu/xhmm/download.shtml). ${ }^{12}$ Quality control measures were calculated using Picard Tools (http://sourceforge.net/projects/picard/files/). Samples were sequenced to a mean coverage of $224 \times$. Three samples were removed from the analysis for having $>10 \%$ of targets with $0 \%$ coverage or $<50 \%$ of targets with $>10 \times$ coverage.

To identify all single-nucleotide variants, small and mediumsize indels, and large genomic rearrangements, variants were filtered to remove synonymous missense variants and intronic variants. Variants were removed from analysis if the alternative allele frequency was less than 0.2 and the total number of reads was less than 20. All other insertions, deletions, nonsense variants, and splicing variants were retained for further analysis. Variants were kept for further analysis if found at an allele frequency of $<0.1 \%$ in both the ESP6500 (http://evs.gs.washington. edu/EVS/) and 1000 Genomes (http://www.1000genomes.org/ data) databases. Variants were analyzed if found at $0.1-1 \%$ allele frequency and previously reported to be a breast cancer susceptibility variant. Splicing variants were analyzed with Skippy (http://research.nhgri.nih.gov/skippy/) and PupaSuite (http:// docs.bioinfo.cipf.es/projects/pupasuite/wiki/Prioritization documentation). ${ }^{10,13}$ All variants were visually inspected in the Integrative Genomics Viewer (http://www.broadinstitute.org/ software/igv/log-in). ${ }^{10}$

\section{Variant classification}

To classify variants into a five-tiered system, a pipeline was developed that integrated posterior probability of pathogenicity data (when available), publically available database calls, protein position of the variant in a functional domain, and in silico analysis ${ }^{10}$ of the effect of the variant on conservation (with GERP, Siphy, and PhyloP) and functionality (with SIFT, Polyphen2, LRT, MutationTaster, and MutationAssessor) (Supplementary Table S1 online). Specifically, variants were first assigned as a variant of uncertain significance (VUS) if (i) the posterior probability of pathogenicity is $>0.0518$ as recorded in the gene's locus-specific database (LSDB) if available or (ii) if the variant was not found in the EVS6500, 1000 Genomes, and dbSNP databases, if an LSDB was not available. If these conditions were not met, then the variant was classified as a likely benign variant (i.e., if (i) the posterior probability of pathogenicity is $<0.0518$ as recorded in the LSDB, if available, or (ii) if the variant was found in the EVS6500, 1000 Genomes, or dbSNP databases, if an LSDB was not available). Exceptions were made for known pathogenic variants found in the EVS6500, 1000 Genomes, and dbSNP databases (i.e., CHEK2 c.1100delC). For the VUSs, variants were upgraded to deleterious variant if called pathogenic by two or more databases: HGMD (http:// www.hgmd.org/), ClinVar (https://www.ncbi.nlm.nih.gov/clinvar/), and the LSDB of the gene (http://www.hgvs.org/dblist/ glsdb.html). VUSs were upgraded to likely deleterious variants if at least four of the following five features ("D points") 
indicated pathogenicity of the variant: (i) position of variant in a biologically important functional domain of the protein known to harbor pathogenic mutations; (ii) pathogenic call in one database (HGMD, ClinVar, and the LSDB of the gene); (iii) a normalized conservation score of $>2$ (maximum 3); (iv) a normalized functional score of $>4$ (maximum 5); and (v) reported nonfunctional in a published in vitro assay. The normalized conservation score was calculated by normalized conservation score $=($ GERPScore $/ x)+($ PhyloPScore $/ x)+($ SiPhyScore $/ x)$, where $x$ is the maximum score for each caller in the data set. The normalized functional score was calculated by normalized functional score $=(1-$ SIFTScore $)+$ PP2HDIVScore $+(1-$ LRTScore) + MutTasterScore + (MutAssessorScore/ $x$ ), where $x$ is the maximum score for each caller in the data set. If the normalized functional score was between 3 and 4 , then the variant was given one D point if the AlignGVGD score (http://agvgd. iarc.fr/agvgd_input.php $)^{14}$ was C55 or C65 or if the CONDEL score (http://agvgd.iarc.fr/agvgd_input.php) ${ }^{15}$ was “D”. For the likely benign variants, these variants were upgraded to VUSs if at least two features ("D points," listed above) indicated pathogenicity of the variant. Likely benign variants were downgraded to benign variants if called a single-nucleotide polymorphism by more than two databases (HGMD, ClinVar, dbSNP, and the LSDB of the gene).

\section{Validation of pipeline}

To determine the efficiency and accuracy of our sequencing platform and bioinformatics and variant classification pipeline, we analyzed samples with variants identified by clinical sequencing in BRCA1, BRCA2, MSH2, or PALB2; these included two nonsense mutations, four indels, two large genomic rearrangements, and 34 single-nucleotide variants.

Table 1 Clinical and pathological characteristics of patients

\begin{tabular}{|c|c|c|c|c|c|}
\hline Characteristic & $\begin{array}{c}\text { Study } \\
\text { population } \\
(n=278)\end{array}$ & $\begin{array}{c}\text { Mutation } \\
\text { positive }^{a} \\
(n=31)\end{array}$ & $\begin{array}{c}\text { VUS } \\
\text { positive }^{\text {b }} \\
(n=55)\end{array}$ & $\begin{array}{c}\text { Mutation/VUS } \\
\text { negative }^{c} \\
(n=192)\end{array}$ & $\begin{array}{l}\text { Mutation positive } \\
\text { vs. the rest of the } \\
\text { population }\end{array}$ \\
\hline & & & & & $P$ value \\
\hline Average age of onset of $B C$ & $34(20-39)$ & $34(23-39)$ & $34(24-39)$ & $34(20-39)$ & NS \\
\hline \multicolumn{6}{|l|}{ Self-reported race/ethnicity, $n$ (\%) } \\
\hline Othere & $12(4)$ & 0 & $5(9)$ & $7(4)$ & NS \\
\hline Not reported & $10(4)$ & $2(7)$ & $2(4)$ & $7(4)$ & NS \\
\hline Ashkenazi Jewish & $27(10)$ & $6(19)$ & $3(5)$ & $18(9)$ & NS \\
\hline Non-Jewish & $234(84)$ & $23(74)$ & $51(93)$ & $160(83)$ & NS \\
\hline \multicolumn{6}{|l|}{ Personal cancer history, $n$ (\%) } \\
\hline Breast cancer & $188(68)$ & $25(81)$ & $35(64)$ & $129(67)$ & NS \\
\hline Breast cancer age $<40$ years & $76(27)$ & $9(29)$ & $15(27)$ & $52(27)$ & NS \\
\hline Bilineal breast cancer & $34(12)$ & $7(23)$ & $5(9)$ & $22(11)$ & 0.08 \\
\hline \multicolumn{6}{|l|}{$B R C A 1 / 2$ prediction models (\%) } \\
\hline Penn II prior probability & 21 & 27 & 20 & 19 & 0.04 \\
\hline BOADICEA & 15 & 29 & 13 & 14 & 0.005 \\
\hline \multicolumn{6}{|l|}{ Pathological } \\
\hline Ductal carcinoma in situ, $n(\%)$ & $23 / 278(8)$ & 4/31 (13) & $7 / 55(13)$ & $12 / 192(6)$ & NS \\
\hline$E R+$ invasive $B C$ & $147 / 214(69)$ & 19/22 (86) & $29 / 44(66)$ & 99/148 (67) & 0.09 \\
\hline Her2+ invasive BC & 49/175 (28) & $7 / 20(35)$ & $10 / 30(33)$ & $32 / 125(26)$ & NS \\
\hline
\end{tabular}

BC, breast cancer; ER, estrogen receptor; NA, not available; NS, not significant; VUS, variant of uncertain significance.

alncluding 30 patients with deleterious and likely deleterious mutations and one MUTYH compound heterozygote. 'اncluding patients with a VUS only or a single MUTYH variant. Including patients with no deleterious variants, likely deleterious variants, or VUSs. ${ }^{d}$ Comparisons were made using a two-tailed Fisher's exact test, except for comparisons of age, Penn II scores, and BOADICEA scores, which used a two-tailed, type 2 Student's $t$ test. Comparisons were also run for mutation-positive versus mutation-negative and VUS negative (excluding the VUS-positive patients) and all $P$ values were consistent. eOther includes individuals of Asian descent (4), Hispanics/Latinos (6), and individuals reporting more than one race (2). ${ }^{\dagger}$ Any malignancy, excluding nonmelanoma skin cancer. 
Of the 42 known variants, $100 \%$ were identified and correctly classified. For each identified deleterious and likely deleterious variant in a study sample, a separate stock aliquot of the patient's DNA sample from the aliquot used for MPS was used for Sanger sequencing of the genomic region containing the variant. Primers were developed using National Center for Biotechnology Information Primer Design software (Bethesda, MD), and polymerase chain reaction products were generated with Platinum Taq polymerase (Life Technologies, Carlsbad, CA).

\section{Statistical analysis of clinicopathological variables}

Statistical comparisons were made regarding the frequency of patients with certain clinical or pathological features within groups of patients as determined by variant status using a twotailed Fisher's exact test. Statistical comparisons of age, Penn II scores, and Breast and Ovarian Analysis of Disease Incidence and Carrier Estimation Algorithm (BOADICEA) scores between groups of patients depending on variant status were performed using a two-tailed, type 2, Student's $t$ test. Comparisons were run for deleterious/likely deleterious variant-positive versus deleterious/likely deleterious variant-negative patients (including the VUS-positive patients in the latter group) and deleterious/likely deleterious variant-positive versus deleterious/likely deleterious variant- and VUS-negative patients (excluding the VUS-positive patients from both groups).

\section{RESULTS}

Characteristics of the early-onset breast cancer study population studied are shown in Table 1 . Of the 278 patients, 169 $(61 \%)$ had at least one variant found at $<0.1 \%$ allele frequency in control public databases. After variant classification, 86 patients $(31 \%)$ were found to have at least one deleterious variant, likely deleterious variant, or VUS (Figure 1). Thirty-one patients $(11 \%)$ overall were found to carry a total of 34 deleterious or likely deleterious variants, 53 patients (19\%) had 57 VUSs (including 49 with a VUS only), and 6 patients $(2.2 \%)$ were heterozygous for MUTYH variants.

Seven patients were found to have deleterious or likely deleterious variants in a high-penetrance cancer susceptibility gene (Figure 1 and Table 2). Two patients were found to carry a known pathogenic TP53 mutation (Figure 2a,b). Two patients, including one African American, were found to carry likely deleterious variants in TP53. One patient was found to have a large genomic rearrangement deleting exon 5 of $\mathrm{MSH} 2$, leading to an in-frame deletion of 65 amino acids of the DNA interacting domain of MSH2. A patient with a history of both early-onset breast cancer and sarcoma was found to carry a known pathogenic missense mutation in CDKN2A. Finally, one patient with a personal history of early-onset colon cancer and two primary breast cancers was found to be a compound heterozygote for a known pathogenic mutation and a likely deleterious variant in MUTYH.

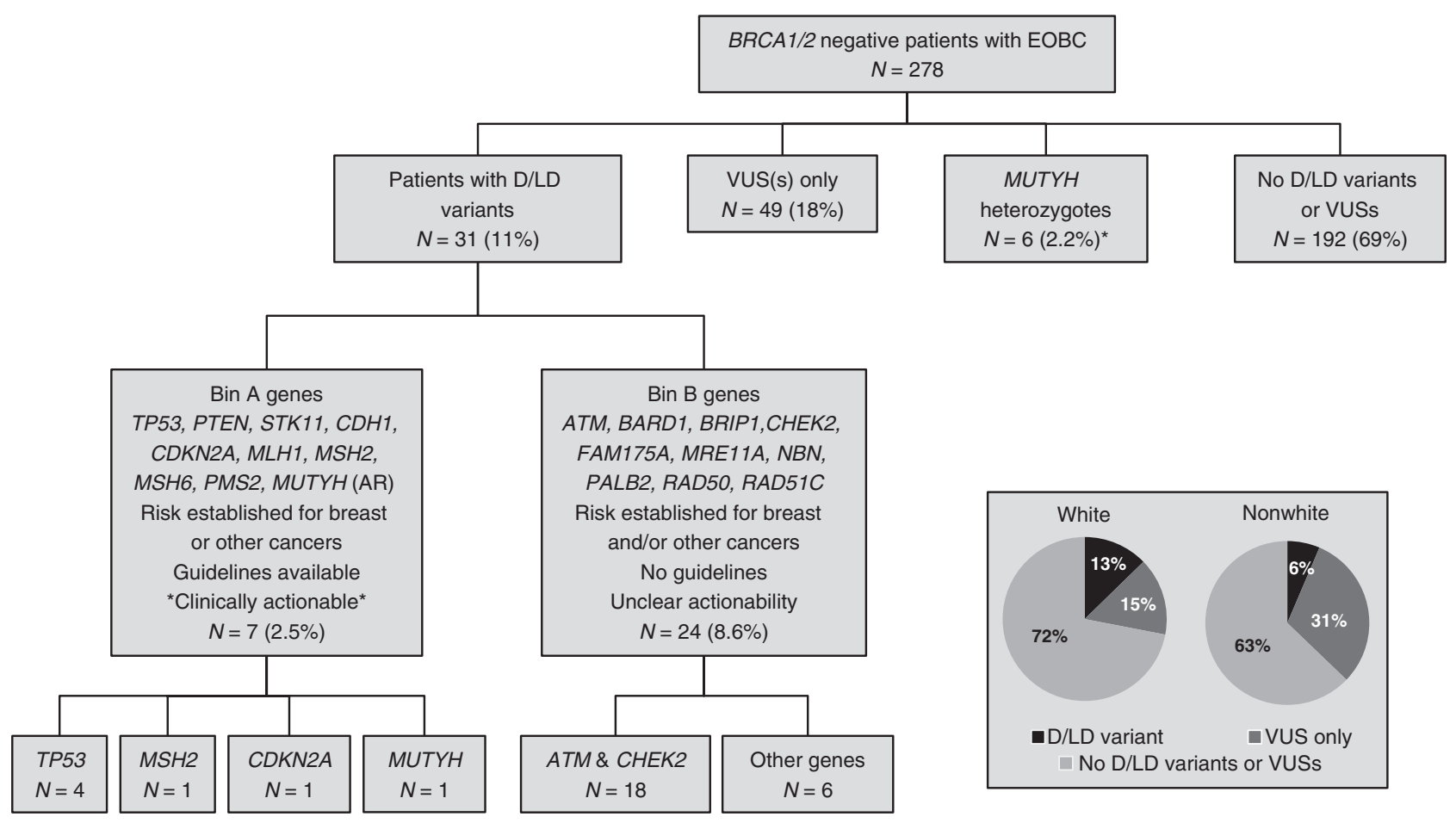

Figure 1 Variants identified by multiplex panel testing of 278 patients with early-onset breast cancer. Germ-line DNA from 278 BRCA1/2-negative patients with early-onset breast cancer (EOBC) was isolated and subjected to massively parallel sequencing using a custom capture for the indicated genes in bin $A$ and bin B. Sequencing data were analyzed with a custom bioinformatics pipeline and deleterious variants were called into classes (B, benign; $D$, deleterious; LB, likely benign; LD, likely deleterious; and VUS, variant of uncertain significance). Inset: Proportion of patients self-reported as "white" or "nonwhite" with deleterious or likely deleterious variants, VUSs only, or no reportable deleterious or likely deleterious variants or VUSs. The MUTYH heterozygous carriers included three patients heterozygous for a deleterious variant and three patients heterozygous for a VUS. 
Table 2 Characteristics of patients with deleterious or likely deleterious variants

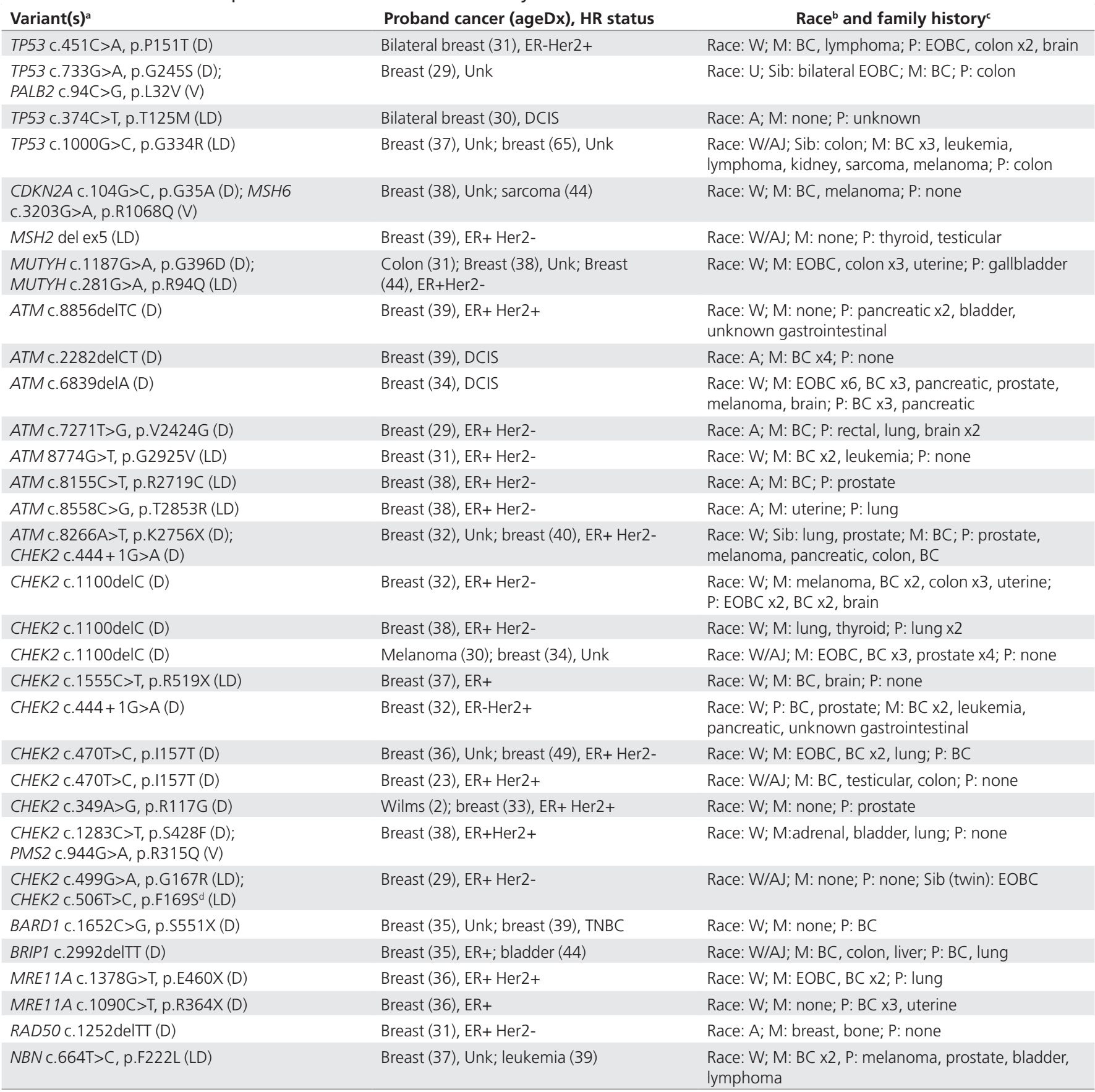

AgeDx, age of diagnosis; DCIS, ductal carcinoma in situ; ER, estrogen receptor; HR, hormone receptor; IGV, Intergrative Genomics Viewer; TNBC, triple negative breast cancer; Unk, unknown.

${ }^{a} \mathrm{D}$, deleterious variant; LD, likely deleterious variant; $\mathrm{V}$, variant of unknown significance (VUS). The method of variant classification is described in the Methods section. Data supporting call for missense variants is provided in Supplementary Table 1. 'bW, white/Caucasian; A, African American; U, unknown; AJ, Ashkenazi Jewish descent. ' $\mathrm{M}$, cancers found on the maternal side; $\mathrm{P}$, cancers found on the paternal side; Sib, cancers found in siblings; EOBC, early-onset (age $<40$ years) breast cancer; $\mathrm{BC}$, breast cancer. ${ }^{\mathrm{d} T h e}$ two CHEK2 mutations were shown to be in trans by analysis of 250 sequencing reads in IGV.

Twenty-four patients were found to have deleterious or likely deleterious variants in genes in which mutations have been associated with a moderate risk of breast cancer. The majority of deleterious or likely deleterious variants in moderatepenetrance genes were found in ATM and CHEK2 (Figure 1 and Table 2). Single deleterious or likely deleterious variants were found in ATM in seven patients and in CHEK2 in nine patients. One patient was found to carry deleterious variants in both ATM and CHEK2; of note, both variants were also found in the patient's brother, who had early-onset prostate cancer (Figure 2c). In addition, one patient was found to carry two likely deleterious variants in trans in CHEK2. The remaining 
six patients had deleterious variants in MRE11A (2), BARD1 (1), BRIP1 (1), NBN (1), and RAD50 (1). Twenty-seven patients carried a VUS in a high-penetrance cancer susceptibility gene, and three of those patients also had a deleterious or likely deleterious variant. Nine patients were found to have a single VUS in BRCA1 or BRCA2, 3 patients had a VUS in TP53, and 12 patients had a VUS in MLH1, MSH2, MSH6, or PMS2; no VUSs were found in CDH1, CDKN2A, STK11, or PTEN. Three additional patients each carried two VUSs in a highpenetrance cancer susceptibility gene. Twenty-six patients were found to have VUSs in moderate-penetrance cancer susceptibility genes, ATM, BRIP1, CHEK2, FAM175A, MRE11A, NBN, PALB2, RAD50, and RAD51C; no VUSs were found in BARD1. Finally, six patients carried a single deleterious variant or VUS in MUTYH (Figure 1). Three patients were heterozygous for the same known pathogenic MUTYH mutation and three were heterozygous for VUSs in MUTYH.

The proportion of patients found to have a clinically reportable variant varied by race, such that $28 \%$ of self-reported white patients were found to have at least one reportable variant versus $37 \%$ of nonwhite patients (Figure 1; $P=$ not significant). The proportion of patients with a deleterious or likely deleterious variant did not vary significantly between white and nonwhite patients ( 13 vs. $6 \%$; $P=$ not significant). The proportion of nonwhite patients found to carry a VUS was statistically significantly higher than the proportion of white patients (31 vs. $15 \%$; $P=0.01$ ). Of the 66 African-American patients, $7.5 \%$ carried a deleterious or likely deleterious variant, which was not statistically significantly different than the proportion of white patients who carried a deleterious or likely deleterious variant. Of the 27 Ashkenazi Jewish individuals, $22 \%$ were found to have a deleterious or likely deleterious variant, as compared with $10 \%$ of the 234 non-Ashkenazi Jewish individuals ( $P=$ not significant).

In comparison with deleterious or likely deleterious variantnegative patients, there was a statistically significant increase in the rate of second primary malignancies (excluding nonmelanoma skin cancers; Table $1 ; 19$ vs. $6 \% ; P=0.02$ ) in the deleterious or likely deleterious variant-positive patients. In addition, there was a trend toward a higher rate of a bilineal family history of breast cancer in deleterious or likely deleterious variant-positive versus variant-negative patients (23 vs. $11 \% ; P=0.08)$. The Penn II $B R C A 1 / 2$ prior probability score ${ }^{16}$ was statistically significantly higher $(27$ vs. $19 \% ; P=0.04)$ in deleterious or likely deleterious variant-positive patients versus deleterious or likely deleterious variant-negative individuals, as was the BOADICEA ${ }^{17}$ score ( 29 vs. $14 \% ; P=0.005$ ).

Only 3 of the 22 patients with deleterious or likely deleterious variants had estrogen receptor (ER)-negative invasive breast cancer (Table 1, 14\%); 1 had triple-negative breast cancer (BARD1 p.S551X) and 2 had ER-negative Her2+ breast cancer (TP53 p.P151T and CHEK2 c.444+1A>G). By contrast, $33 \%$ of the patients with no deleterious or likely deleterious variant (with or without a VUS) had ER-negative invasive breast cancer $(P=0.09)$. Seven of the 20 patients (35\%) with a deleterious or likely deleterious variant had
Her $2+$ breast cancer versus $26 \%$ of the patients with no deleterious or likely deleterious variant (with or without a VUS; $P=$ not significant). Finally, deleterious or likely deleterious variants were found in $13 \%$ of the patients with ductal carcinoma in situ, in $11 \%$ of the 116 patients with node-positive invasive cancer, and in $11 \%$ of the 130 patients with node-negative invasive breast cancer. The stage distribution was similar between deleterious or likely deleterious variant-positive and variant-negative patients.

\section{DISCUSSION}

Using MPS for 22 genes previously associated with cancer susceptibility, we found that $31 \%$ of $B R C A 1 / 2$-negative patients with early-onset breast cancer and no family history of ovarian cancer have a clinically reportable variant, of which one-third were deleterious or likely deleterious variants. However, clinical guidelines exist for the management of cancer risk in only $2.5 \%$ of the patients, those found to have deleterious or likely deleterious variants in TP53, CDKN2A, MSH2, and the MUTYH double heterozygote. Currently, there are no standard-of-care clinical guidelines for the management of cancer risk in the $10 \%$ of women with single mutations in a moderate-penetrance cancer susceptibility gene and MUTYH. Even greater clinical uncertainty exists for the $19 \%$ of patients who were found to carry VUSs.

Multiplex panel MPS-based mutation detection accurately identifies patients with mutations in genes leading to inherited cancer predisposition ${ }^{18}$ and has been used successfully to identify the spectrum of variants in single populations of patients with colon, ovarian, and uterine cancers. ${ }^{19-21}$ Recently, studies have reported findings using multiplex panels in heterogeneous groups of $B R C A 1 / 2$-negative patients, either in randomly selected $^{22,23}$ or consecutive ${ }^{24}$ patients from high-risk genetics clinics or in all patient samples submitted to commercial testing laboratories. ${ }^{23,25}$ Excluding monoallelic MUTYH carriers because the associated cancer risks are controversial, ${ }^{26}$ these studies of predominantly white individuals found that between 3.4 and $9.5 \%$ of $B R C A 1 / 2$-negative patients carried deleterious or likely deleterious variants in panel genes. ${ }^{22-25}$ We found a deleterious or likely deleterious variant rate of $11 \%$ using a custom 22-gene panel in a well-characterized group of 278 early-onset breast cancer patients, including 66 African Americans, consistent with an increased likelihood of finding cancer susceptibility mutations in a younger affected patient population. We found that $2.2 \%$ were heterozygous MUTYH carriers, similar to the LaDuca et al. ${ }^{25}$ study rate of $1.7 \%$ and the reported population carrier frequency of MUTYH mutations of $1.1 \%$ (range: $0-2 \%)^{27}$

Our variant classification algorithm found a 19\% VUS rate in the early-onset breast cancer patients using a pipeline integrating multiple data sources. Kurian et al. used only two in silico variant calling programs and population frequency data to analyze variants and reported a much higher $88 \%$ VUS rate. Our VUS rate is consistent with that of LaDuca et al. (20\%) identified using Ambry's proprietary variant calling program, 
a

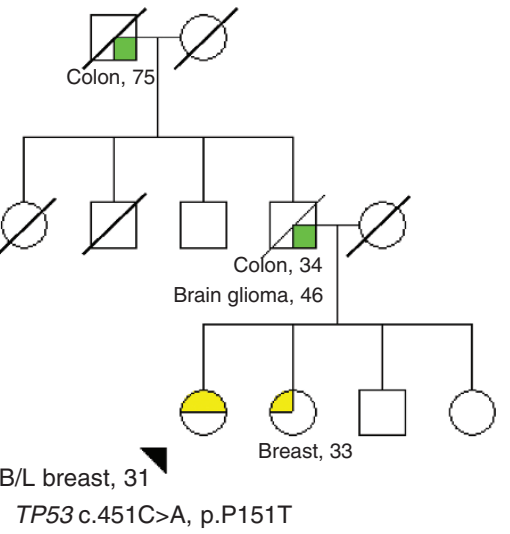

Massively parallel sequencing data: Alternate/total reads: $47 / 92$

Allele frequency: 0.51

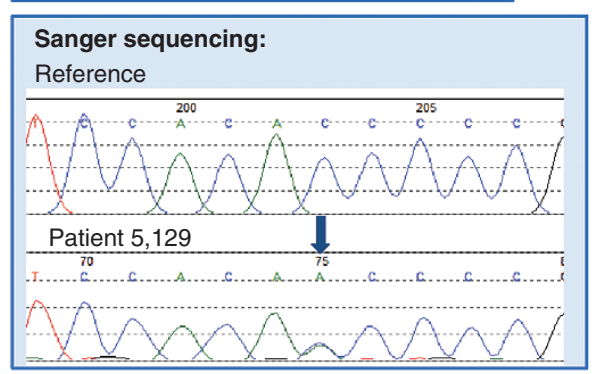

C

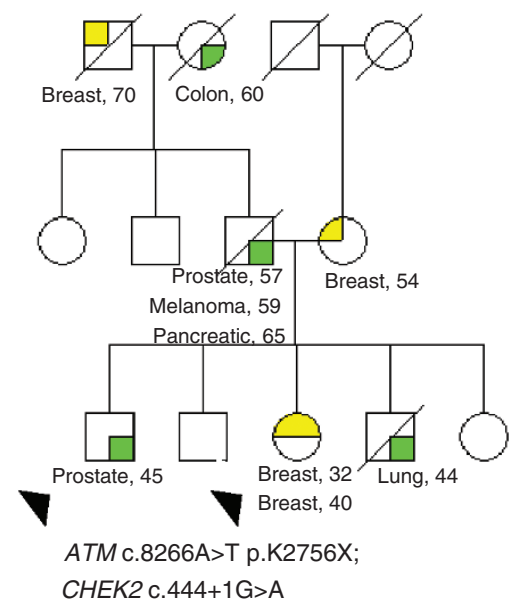

Massively parallel sequencing data: Alternate/total reads: $7 / 22 ; 5 / 28$

Allele frequency: $0.31 ; 0.18$ b

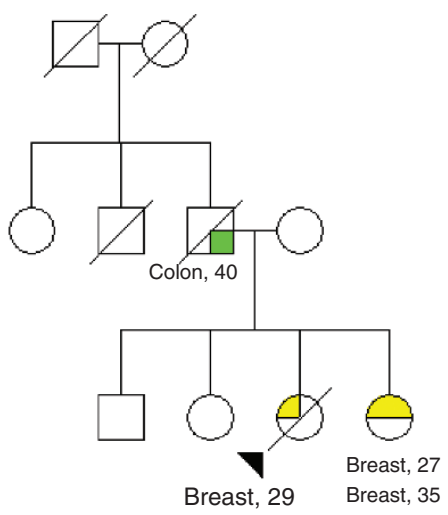

TP53 c.733G>A, p.G245S

Massively parallel sequencing data:

Alternate/total reads: $19 / 43$

Allele frequency: 0.44
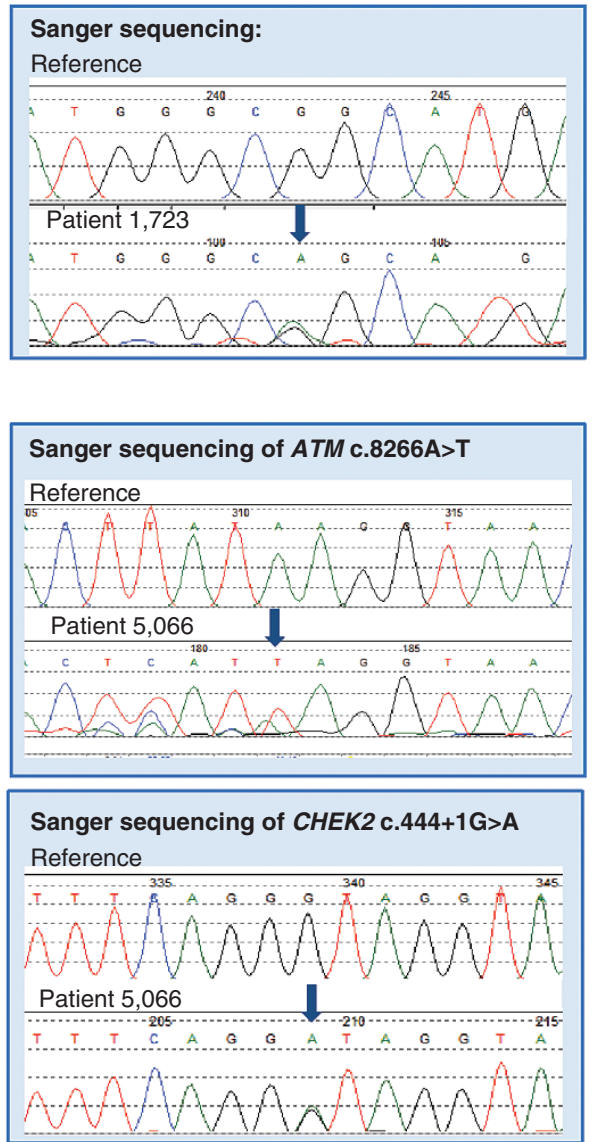

Figure 2 Representative family histories and sequencing data for three probands with identified mutations. (a) Patient 5,129: TP53 C.451C $>A$, p.P151T found by massively parallel sequencing and confirmed by Sanger sequencing. (b) Patient 1,723: TP53 c.733G>A, p.G245S found by massively parallel sequencing and confirmed by Sanger sequencing. (c) Patient 5,066: ATM c.8266A>T p.K2756X and CHEK2 c.444+1G>A found by massively parallel sequencing and confirmed by Sanger sequencing in both the proband and her brother (arrows).

although lower than that of Tung et al. ${ }^{23}(42 \%)$, identified using Myriad's variant calling method. Given that VUSs cause confusion and anxiety for both patients and practitioners, incorporating various data sources to support calls and exploring novel variant classification methods will be increasingly necessary going forward.
In our study, we found that seven patients (2.5\%) carried clinically reportable variants in TP53. Regarding the four individuals with TP53 deleterious or likely deleterious variants, two had family histories meeting Chompret criteria, one was diagnosed at 30 years of age with bilateral breast cancer, and one had a family history of late-onset sarcoma and multiple late-onset bilateral breast 
cancer cases; all were ascertained before 2007. No mutations were found in the genes associated with other well-characterized cancer susceptibility syndromes: PTEN, STK11, and CDH1. Many of the patients in this study population were reviewed in a genetics conference at a tertiary-care institution where there is a high index of awareness for these phenotypes, and patients with known mutations in these genes were excluded from the present study. Their mutation rates may differ in unselected populations.

With regard to other high-risk cancer susceptibility genes, one patient with a family history of melanoma was found to have a mutation in $C D K N 2 A$; excess breast cancer has been described in families with CDKN2A mutations. ${ }^{28}$ One patient was found to have a likely deleterious variant in $\mathrm{MSH} 2$ and one patient was a compound heterozygote for a MUTYH pathogenic mutation and a likely deleterious variant; the breast cancer risks associated with mutations in MUTYH and the mismatch repair genes such as $\mathrm{MSH} 2$ are controversial. ${ }^{29,30}$ It is possible that these mutations did not contribute to the development of breast cancer in these individuals. Further study of the breast cancer risks associated with these gene mutations is needed. These data highlight the importance of determining the clinical management of individuals identified to have mutations by multiplex panel testing in genes not classically associated with the patient's phenotype or pedigree.

Regarding moderate-risk breast cancer susceptibility genes, we found ATM mutations in 2.9\% $(n=8)$, CHEK2 founder mutations (1100delC, I157T, and c. $444+1 \mathrm{G}>\mathrm{A})$ in $2.5 \%(n=7)$, and other CHEK2 mutations in $1.4 \%(n=4)$ of patients. In addition, we found two patients with $M R E 11 A$ mutations and single patients with mutations in $B A R D 1, B R I P 1, N B N$, or RAD50. Interestingly, we did not identify any patients with $P A L B 2$ or $R A D 51 C$ mutations. It is possible that the ethnic diversity of our population ( $28 \%$ nonwhite) is responsible for the variability in mutation frequency between our and other studies. ${ }^{31-35}$ Our study demonstrates that mutations in individual moderatepenetrance genes outside of ATM and CHEK2 are likely very infrequent in patients with early-onset breast cancer.

There are a number of important limitations to our study. Our study design excluded individuals with a personal or family history of ovarian cancer, and it is possible that such earlyonset breast cancer patients will have a different spectrum of mutations. Our study also did not include genes recently proposed to contribute to breast cancer susceptibility, such as $B L M,{ }^{36}$ FANCC, ${ }^{36}$ and $X R C C 2,{ }^{37}$ or ovarian cancer susceptibility, such as $R A D 51 D,{ }^{38}$ and mutations in these genes could be present in our study population. MPS approaches have limitations in the identification of large genomic rearrangements; therefore, these types of variants could still be present in our patient population. Finally, because the majority of patients in the study had a family history of breast cancer and were ascertained through two health systems and affiliated hospitals, our findings may not be generalizable to patients with early-onset breast cancer ascertained through population-based studies.

Overall, our results suggest that at least $11 \%$ of BRCA1/2negative patients with early-onset breast cancer may have a causative mutation in high-penetrance or moderate-penetrance genes found on multiplex panel testing. A higher incidence of other malignancies may occur in early-onset breast cancer patients with these mutations, and further study of these risks in larger populations could allow for more rational decision making regarding cancer screening and medical and/or surgical preventive treatments for these patients, ${ }^{3}$ for example, prophylactic contralateral mastectomy at the time of a breast cancer diagnosis. In addition, it is now understood that the tumors in BRCA $1 / 2$ carriers show increased sensitivity to poly ADP ribose polymerase (PARP) inhibitors and platinum agents due to synthetic lethality. ${ }^{39}$ Given that many of the other cancer susceptibility genes studied here also play a role in doublestranded DNA repair, it is possible that tumors of carriers of some of these other gene mutations may also show increased sensitivity to these agents. ${ }^{40}$

Although our sample size was too limited to define the breast and nonbreast cancer risks for family members of individuals with mutations in moderate-penetrance genes, the Penn II and BOADICEA model prior probability scores were statistically significantly higher in deleterious or likely deleterious variant-positive patients, and this may reflect the stronger family histories of breast and/or other cancers in patients with deleterious mutations. Additional studies are needed to determine if true negative family members of those with mutations in the genes studied here can be counseled that they are at population risk for breast and other gene specific cancers, as is the case for $B R C A 1 / 2{ }^{3}$

Our results highlight the critical need for large consortia to delineate the expected mutation rates, penetrance, and associated cancer risks for moderate-risk genes found on cancer susceptibility genetic testing panels in well-defined clinical populations, keeping in mind the relatively lower penetrance of some of these mutations and the possibility for segregation of multiple risk alleles. In addition, consortia will be needed to pool data to study and develop clinical recommendations for patients carrying these mutations and their family members.

\section{SUPPLEMENTARY MATERIAL}

Supplementary material is linked to the online version of the paper at http://www.nature.com/gim.

\section{ACKNOWLEDGMENTS}

The authors acknowledge the funding sources for this work: the Department of Defense, the National Institutes of Health, the American Association for Cancer Research, the Breast Cancer Research Foundation, the Rooney Family Foundation, the Basser Center for BRCA Research at the University of Pennsylvania, the MacDonald Cancer Risk Evaluation Program, the Susan G. Komen Foundation, and the CURE (Commonwealth Universal Research Enhancement) Program (K.L.N.). The work of K.N.M. was funded by a Department of Defense Breast Cancer Research Program Postdoctoral Fellowship, the National Institutes of Health, and an American Association for Cancer Research Translational Research Fellowship. The work of A.R.B. was funded by the National Institutes of Health, the Rooney Family Foundation, and the Mac- 
Donald Cancer Risk Evaluation Program. The work of S.M.D. was funded by the Rooney Family Foundation, the Basser Center for BRCA Research at the University of Pennsylvania, the MacDonald Cancer Risk Evaluation Program, and the Susan G. Komen Foundation. The authors express their sincere gratitude to the patients who provided samples for this research and the health-care professionals who recruited the patients included in this study.

The Pennsylvania Department of Health specifically disclaims responsibility for any analyses, interpretations, or conclusions. Views and opinions of, and endorsements by, the authors do not reflect those of the US Army or the Department of Defense. All work contained in this article is original. This study was presented in part at the 2013 annual meeting of the American Association for Cancer Research and as an oral presentation in a Clinical Science Symposium at the 2014 annual meeting of the American Society of Clinical Oncology.

\section{DISCLOSURE}

The authors declare no conflict of interest.

\section{REFERENCES}

1. Sundquist M, Thorstenson S, Brudin L, Wingren S, Nordenskjöld B. Incidence and prognosis in early onset breast cancer. Breast 2002;11:30-35.

2. Daly MB, Axilbund JE, Buys S, et al.; National Comprehensive Cancer Network. Genetic/familial high-risk assessment: breast and ovarian. J Nat/ Compr Canc Netw 2010;8:562-594.

3. Maxwell KN, Domchek SM. Cancer treatment according to BRCA1 and BRCA2 mutations. Nat Rev Clin Oncol 2012;9:520-528.

4. Maxwell KN, Domcheck SM. Familial breast cancer risk. Curr Breast Cancer Rep 2013;5:170-182.

5. Shendure J, Lieberman Aiden E. The expanding scope of DNA sequencing. Nat Biotechnol 2012;30:1084-1094.

6. Stadler ZK, Schrader KA, Vijai J, Robson ME, Offit K. Cancer genomics and inherited risk. J Clin Oncol 2014;32:687-698.

7. Biesecker LG, Burke W, Kohane I, Plon SE, Zimmern R. Next-generation sequencing in the clinic: are we ready? Nat Rev Genet 2012;13:818-824.

8. Domchek SM, Bradbury A, Garber JE, Offit K, Robson ME. Multiplex genetic testing for cancer susceptibility: out on the high wire without a net? J Clin Oncol 2013;31:1267-1270.

9. Zheng $Y$, Ogundiran TO, Falusi AG, et al. Fine mapping of breast cancer genome-wide association studies loci in women of African ancestry identifies novel susceptibility markers. Carcinogenesis 2013;34:1520-1528.

10. Pabinger $S$, Dander $A$, Fischer $M$, et al. A survey of tools for variant analysis of next-generation genome sequencing data. Brief Bioinform 2014;15:256-278.

11. McKenna A, Hanna M, Banks E, et al. The Genome Analysis Toolkit: a MapReduce framework for analyzing next-generation DNA sequencing data. Genome Res 2010;20:1297-1303.

12. Fromer M, Moran JL, Chambert $\mathrm{K}$, et al. Discovery and statistical genotyping of copy-number variation from whole-exome sequencing depth. Am J Hum Genet 2012;91:597-607.

13. Woolfe A, Mullikin JC, Elnitski L. Genomic features defining exonic variants that modulate splicing. Genome Biol 2010;11:R20.

14. Tavtigian SV, Oefner PJ, Babikyan D, et al.; Australian Cancer Study; Breast Cancer Family Registries (BCFR); Kathleen Cuningham Foundation Consortium for Research into Familial Aspects of Breast Cancer (kConFab). Rare, evolutionarily unlikely missense substitutions in ATM confer increased risk of breast cancer. Am J Hum Genet 2009;85:427-446.

15. González-Pérez A, López-Bigas N. Improving the assessment of the outcome of nonsynonymous SNVs with a consensus deleteriousness score, Condel. Am J Hum Genet 2011;88:440-449.

16. Lindor NM, Johnson KJ, Harvey $\mathrm{H}$, et al. Predicting BRCA1 and BRCA2 gene mutation carriers: comparison of PENN II model to previous study. Fam Cancer 2010;9:495-502.

17. Lee AJ, Cunningham AP, Kuchenbaecker KB, Mavaddat N, Easton DF, Antoniou AC; Consortium of Investigators of Modifiers of BRCA1/2; Breast
Cancer Association Consortium. BOADICEA breast cancer risk prediction model: updates to cancer incidences, tumour pathology and web interface. $\mathrm{Br}$ J Cancer 2014;110:535-545.

18. Walsh $\mathrm{T}$, Lee MK, Casadei $\mathrm{S}$, et al. Detection of inherited mutations for breast and ovarian cancer using genomic capture and massively parallel sequencing. Proc Natl Acad Sci USA 2010;107:12629-12633.

19. Pennington KP, Walsh $T$, Lee $M$, et al. BRCA1, TP53, and CHEK2 germline mutations in uterine serous carcinoma. Cancer 2013;119:332-338.

20. Cragun D, Radford C, Dolinsky JS, Caldwell M, Chao E, Pal T. Panel-based testing for inherited colorectal cancer: a descriptive study of clinical testing performed by a US laboratory. Clin Genet 2014;86:510-520.

21. Walsh T, Casadei $\mathrm{S}$, Lee MK, et al. Mutations in 12 genes for inherited ovarian, fallopian tube, and peritoneal carcinoma identified by massively parallel sequencing. Proc Natl Acad Sci USA 2011:108:18032-18037.

22. Kurian AW, Hare EE, Mills MA, et al. Clinical evaluation of a multiplegene sequencing panel for hereditary cancer risk assessment. J Clin Oncol 2014;32:2001-2009.

23. Tung $N$, Battelli $C$, Allen $B$, et al. Frequency of mutations in individuals with breast cancer referred for BRCA1 and BRCA2 testing using next-generation sequencing with a 25-gene panel. Cancer; e-pub ahead of print 3 September 2014

24. Castéra L, Krieger S, Rousselin A, et al. Next-generation sequencing for the diagnosis of hereditary breast and ovarian cancer using genomic capture targeting multiple candidate genes. Eur J Hum Genet 2014;22:1305-1313.

25. LaDuca H, Stuenkel AJ, Dolinsky JS, et al. Utilization of multigene panels in hereditary cancer predisposition testing: analysis of more than 2,000 patients. Genet Med 2014;16:830-837.

26. Win AK, Dowty JG, Cleary SP, et al. Risk of colorectal cancer for carriers of mutations in MUTYH, with and without a family history of cancer. Gastroenterology 2014;146:1208-1211.e1-5.

27. Peterlongo P, Mitra N, Chuai S, et al. Colorectal cancer risk in individuals with biallelic or monoallelic mutations of MYH. Int J Cancer 2005;114:505-507.

28. Borg A, Sandberg T, Nilsson K, et al. High frequency of multiple melanomas and breast and pancreas carcinomas in CDKN2A mutation-positive melanoma families. J Natl Cancer Inst 2000;92:1260-1266.

29. Win AK, Lindor NM, Jenkins MA. Risk of breast cancer in Lynch syndrome: a systematic review. Breast Cancer Res 2013:15:R27.

30. Beiner ME, Zhang WW, Zhang S, Gallinger S, Sun P, Narod SA. Mutations of the MYH gene do not substantially contribute to the risk of breast cancer. Breast Cancer Res Treat 2009;114:575-578.

31. FitzGerald MG, Bean JM, Hegde SR, et al. Heterozygous ATM mutations do not contribute to early onset of breast cancer. Nat Genet 1997;15: 307-310.

32. Cao AY, Huang J, Hu Z, et al. Mutation analysis of BRIP1/BACH1 in BRCA1/ BRCA2 negative Chinese women with early onset breast cancer or affected relatives. Breast Cancer Res Treat 2009;115:51-55.

33. Cao AY, Huang J, Hu Z, et al. The prevalence of PALB2 germline mutations in BRCA1/BRCA2 negative Chinese women with early onset breast cancer or affected relatives. Breast Cancer Res Treat 2009;114:457-462.

34. Ding D, Zhang Y, He X, Meng W, Ma W, Zheng W. Frequency of the CHEK2 $1100 \mathrm{del} C$ mutation among women with early-onset and bilateral breast cancer. Breast Cancer Res 2012;14:401.

35. Foulkes WD, Ghadirian P, Akbari MR, et al. Identification of a novel truncating PALB2 mutation and analysis of its contribution to early-onset breast cancer in French-Canadian women. Breast Cancer Res 2007;9:R83.

36. Thompson ER, Doyle MA, Ryland GL, et al.. Exome sequencing identifies rare deleterious mutations in DNA repair genes FANCC and BLM as potential breast cancer susceptibility alleles. PLoS Genet 2012;8:e1002894.

37. Park DJ, Lesueur F, Nguyen-Dumont T, et al.; Breast Cancer Family Registry; Kathleen Cuningham Foundation Consortium for Research into Familial Breast Cancer. Rare mutations in XRCC2 increase the risk of breast cancer. Am J Hum Genet 2012;90:734-739.

38. Thompson ER, Rowley SM, Sawyer S, et al. Analysis of RAD51D in ovarian cancer patients and families with a history of ovarian or breast cancer. PLoS One 2013;8:e54772

39. Fong PC, Boss DS, Yap TA, et al. Inhibition of poly(ADP-ribose) polymerase in tumors from BRCA mutation carriers. N Engl J Med 2009;361: 123-134.

40. Pennington KP, Walsh $\mathrm{T}$, Harrell Ml, et al. Germline and somatic mutations in homologous recombination genes predict platinum response and survival in ovarian, fallopian tube, and peritoneal carcinomas. Clin Cancer Res 2014;20:764-775. 